\title{
Psoriasis today: experiences of healthcare and impact on quality of life in a major UK cohort
}

\section{Authors}

A.S. Nash $^{1}$, H. McAteer ${ }^{2}$, J. Schofield ${ }^{1}$, R. Penzer ${ }^{3}$, A.K. Gilbert ${ }^{2}$

University of Hertfordshire ${ }^{1}$, The Psoriasis Association ${ }^{2}$, Community Dermatology Specialist Nurse for Norfolk Community Health and Care Trust $^{3}$

Corresponding author: Dr Avril Nash

Centre for Research in Primary and Community Care, University of Hertfordshire, College Lane, Hatfield, AL10 9AB

Tel: 01707284304

Fax: 01707285995

Email: a.s.nash@herts.ac.uk 


\section{Psoriasis today: experiences of healthcare and impact on quality of life in a major UK cohort}

\section{Introduction}

In the UK and Northern Ireland it is estimated that up to $2 \%$ of the population have psoriasis (C.E.M. Griffiths \& Barker, 2010), a visible and, for some, disabling, inflammatory skin disease (Bhosle, Kulkarni, Feldman, \& Balkrishnan, 2006; Poulin et al., 2010; Wahl et al., 2006). Surveys of people coping with long term conditions such as psoriasis can provide valuable insights and should lead to improvements in care. They also establish a baseline against which any future improvements in experience can be measured and evaluated. While a large USA survey of psoriasis patients is now some 13 years old, surveys have been conducted more recently in Canada, France and Europe (Fouere, Adjadj, \& Pawin, 2005; Krueger et al., 2001; Meyer et al., 2010; Poulin et al., 2010; Schmitt-Egenolf, 2006), and a recent UK study focussed on the psychosocial impact of psoriasis (Anstey, McAteer, Kamath, \& Percival, 2012). These, and the more general psoriasis literature, document the difficulties experienced by those with this condition such as soreness, stigmatisation, difficult-to-manage treatment regimes, morbidity, associated co-morbidity, and a negative impact on their quality of life (Fouere et al., 2005; Gottlieb \& Dann, 2009; Krueger et al., 2001; Langley, Krueger, \& Griffiths, 2005; Meyer et al., 2010; Poulin et al., 2010).

Interestingly, one of the European studies suggested that in the UK people with psoriasis $(n=246)$ were far more likely to rate their condition as 'mild' and to have treatments prescribed by their general practitioner when compared to their European 
counterparts from Germany, France, Belgium and the Netherlands (Fouere et al., 2005), suggesting that psoriasis is a condition which is often managed in primary care settings in the UK. The other, larger, European study did not include participants from the UK (Dubertret et al., 2006) and a direct comparison of selfassessment of individuals' psoriasis with the recent UK survey is difficult due to different terminology being used in the two studies: for example 'very active' may not equate to 'severe' (Anstey et al., 2012).

While the recent UK study addressed psychosocial factors associated with psoriasis in some depth, there is a lack of recent research into the healthcare experiences of UK patients. The following is a report on the findings from a postal, paper-based, survey which sought to remedy this and to assess and understand people's experiences of diagnosis of psoriasis, their subsequent healthcare and treatments in the UK and the impact on daily life. The discussion includes consideration of key areas for improvement in care.

\section{Material and Methods}

\section{Materials}

A questionnaire was designed to gather demographic data and information on members' experiences of diagnosis, quality of care, treatment, and healthcare professionals. The design of the questionnaire was patient-association driven and focused on topics that arose regularly through the patient/members' helpline. In addition, a coping and quality of life section was included which drew on issues 
covered in the Dermatology Life Quality Index (DLQI) (Finlay \& Khan, 1994). However, in this instance it was not, nor intended to be, a scale, but a series of discrete questions with a dichotomous response for the purpose of gathering data about the impact of psoriasis on specific aspects of daily life. Participants could respond to two open questions in the survey with comments, but the data were predominantly quantitative. Prior to circulation of the survey, minor amendments to the questionnaire were incorporated following invited patient feedback. The research met the requirements of the Nursing, Midwifery and Social Work Ethics Committee at the University of Hertfordshire.

\section{Sample and Procedure}

Questionnaires, accompanied by an introductory letter and a reply-paid envelope, were sent out to all members of The Psoriasis Association $(\mathrm{N}=2830)$ and 1619 were returned, giving a response rate of $57 \%$. Fifty-five of the returned questionnaires contained insufficient data to merit inclusion, making the final sample size $N=1564$. Completion and return of the survey form were deemed consent to participate in the study and all data were entered anonymously into SPSS.

\section{Results}

Both sexes were equally represented as 751 (48\%) participants were male. Ages ranged from below 10 years to over 80 years old and were classified in age groups. Table 1 presents the distribution of the sample by group together with the mean length of time since participants' diagnosis with psoriasis. The mode age range of 
respondents was $61-70$, representing $29 \%$ of the sample and the percentage of returned survey forms by age group was representative of the membership of The Psoriasis Association.

The length of time that respondents had been affected by the condition ranged from 1 month to 89 years. Naturally their age group had a bearing on this and, while the overall mean length of coping with the condition was $32 \mathrm{y} 11 \mathrm{~m}$, analysis of this by age group indicates that the mean length of experience with psoriasis was approximately equal to half participants' current lifespan.

(Table 1 about here)

Respondents experienced different types of psoriasis and sometimes more than one type. Plaque psoriasis was the most common with 1265 (81\%) participants reporting it. Guttate psoriasis was reported by 211 (13.5\%) respondents, while localised and generalised pustular psoriasis were the least frequently reported, with $89(6 \%)$ and $46(3 \%)$ cases respectively.

In terms of location, the scalp was the most commonly affected body area with 1160 (74\%) reporting it, followed by nails, 725 (46\%), sensitive/flexural areas, 396 (25\%), and the face, 375 (24\%). Additionally, 472 respondents reported having psoriatic arthritis, 337 of whom had received a formal diagnosis (representing $21 \%$ of whole sample). Co-morbidity with psoriasis was common, with just under $70 \%$ reporting other conditions, such as hypertension, diabetes and osteoporosis. 


\section{Diagnosis and referral}

The majority (91\%) of participants had approached their general practitioner (GP) for diagnosis in the first instance, with $25 \%$ already believing they had psoriasis. The remainder sought the advice of a pharmacist (2\%) or other health professional, including chiropodists and dermatologists. Psoriasis was correctly diagnosed initially for 1180 (75\%) participants. However, 300 (19\%) were given an incorrect initial diagnosis and responses on some survey forms suggested that reaching the correct diagnosis could be a lengthy process, exceeding a year in some instances.

In total, 1027 (68\%) of respondents were referred by their GP for specialist care and assessment, primarily to consultant dermatologists. While $70 \%$ of those referrals were made on the initiative of the GP, some 298 (30\%) participants requested referral by their GP. Twenty-three participants (2\%) were initially referred to hospitalbased dermatology specialist nurses; none were referred to dermatology specialist nurses in GPs' surgeries. Of those who attended secondary care, $27 \%$ went on, after specialist consultation, to be referred to the dermatology specialist nurse. Only $49 \%$ of those seen in secondary care were able to re-access the specialist dermatology department without re-referral from their GP if the condition flared. Of the total sample, $465(30 \%)$ had been admitted at some point for in-patient treatment, and two-thirds of these had been treated on a specialist ward. Only 42 respondents (2.6\%) reported having in-patient treatment in the previous 12 months.

\section{Healthcare experiences}


At the point of diagnosis, whether by a GP in primary or a specialist in secondary care, patients expressed some dissatisfaction with the information they received. Fifty-four per cent were not given adequate information about psoriasis and 56\% were not given sufficient information about the treatments available. Seventy-four percent reported that they were not offered different treatment options and many (54\%) did not feel included in the decision-making process about their treatment. Forty-seven per cent would have welcomed more time, support and the opportunity to ask questions, and only $10 \%$ of participants were given contact details of support organisations.

In terms of information and decisions about their prescribed treatment, 1137 (72.7\%) of the respondents felt that they were given enough information about the treatments proposed for them in terms of application, how long it would take to work and potential side effects. Only $64 \%$ felt that they had been given sufficient time to consider the choice of treatment. Whilst these are both comparatively sizeable percentages, in a sample of this magnitude, it means that there is still a considerable number of participants who are less satisfied with the information received and the opportunity to make choices.

Relationships with healthcare professionals were, nonetheless, largely positive. Participants only evaluated those health professionals with whom they came into regular contact and the following percentages relate to the sample size, given in brackets, responding about a specific professional. Rating their relationships as either satisfactory or excellent with their GP $(n=1473)$ were $91 \%$ of respondents; 
with their consultant dermatologist $(n=787), 87 \%$; dermatology specialist nurse $(n=$ 360) $94 \%$; and with their pharmacist $(n=772), 97 \%$.

\section{Treatments and outcomes}

Table 2 presents details of treatments being used by survey participants at the time of the study. Some patients were using up to nine different treatments, and topical treatments represented by far the largest category.

(Table 2 about here)

Respondents were asked about both the severity of their psoriasis and about their expectations of treatments. Mild psoriasis was reported by 506 (32\%), while 722 (46\%) respondents classified it as moderate, and $320(21 \%)$ as severe. Table 3 gives mean ratings of how important participants perceive different outcomes of treatment. With a minimum of 1 (not important) and a maximum of 4 (very important), mean scores over 3 signify 'very important'.

(Table 3 about here)

Mean importance of treatment outcome scores were then assessed by severity group (mild, moderate, severe) and differences in mean were subject to analysis of variance. These were found to differ significantly with $p<.05$, suggesting that those with more severely self-rated psoriasis rated the importance of treatment outcomes more highly. Post hoc (LSD) analysis confirmed this. However, no significant 
differences in means were found between the groups rating their psoriasis as 'moderate' or 'severe' for three options - clearance of visible areas, reduced redness and improvements to general wellbeing - indicating, perhaps, aspects of the condition that should be targeted for treatment in order to benefit most psoriasis patients.

\section{Psychosocial, practical and emotional impacts}

Respondents were asked to indicate how psoriasis had affected their daily life over the previous month and Figure 1 presents a frequency analysis of 'yes' responses.

(Figure 1 about here)

The main negative impacts of psoriasis were associated with coping with treatments, psychosocial functioning, and emotional distress. However, the data also clearly indicated that despite $54 \%$ of respondents experiencing emotional distress, only $13 \%$ of those affected received professional help for it. Negative effects on work, study and promotion were considerably lower, but may be influenced by the age group of the sample as $53 \%$ of participants were over the age of 60 . Indeed, age-related changes in the impact of psoriasis were found for other items (see Table 4) and suggest a lessening impact with age, particularly post-60y.

(Table 4 about here) 
A comprehensive analysis of the rich qualitative data produced by the survey exceeds the scope of this article; however, reference to representative comments by respondents support the above findings. For example, topical treatments were perceived negatively by many patients. They were deemed sticky, messy, difficult and time-consuming, to the extent that they were viewed as 'stressful', 'difficult to maintain' and 'a bigger nuisance than the condition'. Moreover they were perceived by many to be ineffective. On the other hand, there were favourable reports by some for the biological therapies. Respondents indicated that GPs should be better informed, they wanted easier access to specialist dermatology clinics and greater innovation by the NHS with regard to psoriasis treatments.

Comments related to psychosocial factors affirmed the pervasive effects of psoriasis on individual lives. These ranged from the daily restrictions on clothing choices and no longer being able to swim or take part in sports, to the "embarrassment of itching and shedding scales at my desk", and the severe negative impact on confidence, social lives, career choices and life paths. Many acknowledged that stress was a trigger for flare-ups, and this was often associated with the practical limitations imposed by psoriasis or arising from the emotional distress which can accompany psoriasis at any age. As one young woman wrote,

"I feel very isolated living with psoriasis ... I feel like it is taking over my entire body day by day and has reduced my confidence to nil".

Another respondent reported that her young granddaughter told her, “I'm not really frightened of you Granny, but I don't like seeing your hands or feet". 
Men were equally affected by the condition and one explained that, "psoriasis now covers $75 \%$ of my body. I no longer swim. I sunbathe when alone. I have amicably separated from my wife as I am very sensitive about my condition."

Such findings, both quantitative and qualitative, indicate distressing aspects of the condition that do not currently appear to be adequately addressed.

\section{Discussion}

This survey presents a comprehensive assessment of the health experiences of people with psoriasis in the UK. It also offers a further insight into the impact of psoriasis on everyday life. The high response rate in this postal survey is, perhaps, indicative of some of the frustration experienced by psoriasis patients in relation to treatments which are currently available, and of the profound negative psychosocial impact and emotional distress associated with this long-term condition. Selfselection bias is a limitation of the study as participants were both members of the Psoriasis Association and prepared to complete the questionnaire. However, a benefit of sampling through the Psoriasis Association is twofold: access to many who do not regularly attend primary or secondary care for their psoriasis, and information collected in an independent setting.

In many respects the findings tend to support those of surveys from the international literature, including the older USA study (Krueger et al., 2001), particularly in relation to knowledge, psychosocial functioning, emotional distress and dissatisfaction with available treatments. However, in this survey, when compared to the UK cohort in 
the European study (Fouere et al., 2005), fewer Britons were likely to report their condition as 'mild'. This may be due, perhaps, to both the larger sample size in this study and to a far more equitable gender ratio (48\% participants were male compared to only $36 \%$ in the UK cohort in the European study). Respondents in this study were far less likely to report a negative impact of psoriasis on work and career than those in the Anstey et al. (2012) study and, again, context is an important consideration when comparing findings. The Anstey et al. study was an online survey which had a sample with a much younger age bias - median age group 40-44y compared to the median age group of 61-70y in this postal survey. Indeed $7 \%$ respondents in this study were over the age of 80 . As might be expected, this is likely to affect responses to questions about work in the previous month. However, such a comparison may also give some indication as to why some of the negative psychosocial impact of psoriasis appears to lessen with age (Unaeze, Nijsten, Murphy, Ravichandran, \& Stern, 2006).

The research provided an informative insight into the health and healthcare experiences of people with psoriasis and indicated a number of areas that merit further research or improvement. For example, health policy in England and Wales favours the self-management of long-term conditions, regarding the patient as expert (Department of Health, 2011a, 2011b). However, for this to be successful, and for patients to be able to properly manage their condition, it is essential that they are fully informed and involved in making choices about their treatment. Yet, in common with other research findings (Poulin et al., 2010), respondents were aware of being under-informed, particularly by their GPs who, as their title suggests, do not have specialist knowledge of the condition nor of all available treatments. Indeed the 
qualitative data suggested that Psoriasis Association events and newsletters provide much-needed information that is not available to them elsewhere, and yet newlydiagnosed patients were rarely given details of this or other skin disorder support organisations. Moreover, the ability to re-access specialist dermatology services as necessary, as clearly recommended in a 2006 White Paper (Department of Health, 2006b), was not apparent according to this research with only half of the respondents able to do this, thus restricting their access to current opinion and knowledge.

The findings suggest scope for improving the training of primary care health professionals, both GPs and nurses, and for increasing the number of dermatology specialist nurses in primary care settings such as GP surgeries. In particular, the latter could help to address needs identified here by providing appropriate care for those with straightforward, pre-diagnosed stable plaque psoriasis, promoting better patient knowledge and ensuring that support group details are provided to all patients diagnosed with psoriasis. In this way changes at primary care level could help to improve patient care, optimise self-management and facilitate access and reaccess to secondary care for those with hard-to-manage psoriasis.

In addition, current treatments frequently failed to meet patient needs and expectations. Topical treatments were the most commonly prescribed and yet patients found them difficult, unpleasant and time-consuming to manage, and perceived them as ineffective. As the effectiveness of treatments can depend on adherence to correct usage (Ersser, Cowdell, Latter, \& Healy, 2010), the difficulties that patients experience with them can render them ineffective, whether or not they 
have the potential to be effective, and can hinder a successful outcome as far as self-management is concerned. Patients need treatment appropriate to the severity of the condition (Poulin et al., 2010) and realistic treatment regimes from medications that, as indicated by the respondents in this survey, reduce the visible evidence of the condition and improve general wellbeing. Certainly treatments that effectively improve visible appearance would have a positive consequence for wellbeing by allowing those with psoriasis a wider choice of clothing, and enabling them to be more socially active, thus promoting greater confidence. In a similar vein this could also widen access to sporting activities, which is vital given the emerging evidence of a link between psoriasis and other co-morbidities such as cardiovascular disease (Gottlieb \& Dann, 2009).

The findings also highlight a fundamental aspect of psoriasis which continues to be overlooked by healthcare professionals and which, again, would contribute to general wellbeing: the lack of support for psychosocial functioning and emotional distress. For example, the disparity between the high proportion of respondents experiencing psoriasis-related emotional distress (54\%) and those receiving psychological support for it (only $13 \%$ of the $54 \%$ ) clearly indicates a need which is currently not being met. Moreover, strong similarities in responses to those found in the Anstey et al. (2012) study regarding emotional distress and confidence suggest that, unlike some of the psychosocial functioning aspects, the negative impact of psoriasis on mental health and emotional wellbeing does not lessen with time and age. Evidence indicates that psychosocial and emotional support can be empowering, and can improve the condition and maintain those improvements by helping people to cope with stress and the daily hurdles imposed by psoriasis 
(Fortune et al., 2002; C. E. M. Griffiths \& Richards, 2001; Seng \& Nee, 1997). Given that stress is a known trigger for flare-ups, such support could prove crucial in helping to break the vicious circle. However, it is also recognised that resistance by some patients to accepting 'psychological' help has been identified (Fortune et al., 2002) and ways to overcome this may need to be researched and developed.

\section{Conclusions}

There were some encouraging findings in the survey in that relationships with healthcare professionals were, for most respondents, very positive and, concurring with other research (e.g. Unaeze et al., 2006), there appears to be a lessening impact on psychosocial functioning with age, and over time. The introduction and effectiveness of biological treatments were seen as positive, although they only benefitted $11 \%$ of respondents. However, aside from these factors, the experiences of respondents in this survey echo many of those in the 1998 US survey (Krueger et al., 2001) which suggests, disappointingly, that solutions to many of the problems associated with psoriasis have advanced little over the last thirteen years, despite continued research.

The findings of this survey highlight that patients need to be better informed about the condition and treatments, both at diagnosis and over time, so that they are in a position to manage their condition effectively. As suggested, improvements in training and resources at primary care level could help to achieve this. Secondly, topical treatments need to be developed further to become both manageable and effective, and to reflect the patient priorities, identified in the survey, of improving 
Psoriasis today: healthcare and quality of life

visible appearance and promoting well-being. Finally, reports of the negative impact of psoriasis on psychosocial functioning and, in particular, the extent of emotional distress that is currently experienced underscore the urgent need to remedy the lack of formal social and psychological support mechanisms for those with psoriasis. These are key issues which, if addressed or further researched, could provide positive and tangible benefits for people coping with psoriasis. 
Psoriasis today: healthcare and quality of life

\section{Acknowledgements}

The authors would like to thank the members of The Psoriasis Association who participated in this research and Gladys Edwards, former CE of The Psoriasis Association, for her support and contribution at the beginning of the project. We are grateful to Schering-Plough for their educational grant which enabled the research to be carried out. 


\section{References}

Anstey, A., McAteer, H., Kamath, N., \& Percival, F. (2012). Extending psychosocial assessment of patients with psoriasis in the UK, using a self-rateds, webbased survey. Clinical and Experimental Dermatology, 37(7), 735-740. doi: 10.1111/j.1365-2230.2012.04457.x

Bhosle, M. J., Kulkarni, A., Feldman, S. R., \& Balkrishnan, R. (2006). Quality of life in patients with psoriasis. Health Qual Life Outcomes, 4, 35. doi: 1477-7525-435 [pii]

10.1186/1477-7525-4-35 [doi]

Department of Health. (2006b). Our health, our care, our say: a new direction for community services. Norwich.

Department of Health. (2011a). Expert patient. London, UK.

Department of Health. (2011b). Self-management. London UK.

Dubertret, L., Mrowietz, U., Ranki, A., van de Kerkhof, P. C., Chimenti, S., Lotti, T., \& Schafer, G. (2006). European patient perspectives on the impact of psoriasis: the EUROPSO patient membership survey. Br J Dermatol, 155(4), 729-736. doi: 10.1111/j.1365-2133.2006.07405.x

Ersser, S. J., Cowdell, F. C., Latter, S. M., \& Healy, E. (2010). Self-management experiences in adults with mild-moderate psoriasis: an exploratory study and implications for improved support. British Journal of Dermatology, 163(5), 1044-1049. doi: 10.1111/j.1365-2133.2010.09916.x

Finlay, A. Y., \& Khan, G. K. (1994). Dermatology Life Quality Index (DLQI) - a simple practical measure for routine clinical use. Clinical and Experimental Dermatology, 19(3), 210-216.

Fortune, D. G., Richards, H. L., Kirby, B., Bowcock, S., Main, C. J., \& Griffiths, C. E. M. (2002). A cognitive-behavioural symptom management programme as an adjunct in psoriasis therapy. British Journal of Dermatology, 146(3), 458-465.

Fouere, S., Adjadj, L., \& Pawin, H. (2005). How patients experience psoriasis: results from a European survey. Journal of the European Academy of Dermatology and Venereology, 19, 2-6.

Gottlieb, A. B., \& Dann, F. (2009). Comorbidities in patients with psoriasis. Am J Med, 122(12), 1150.e1151-1159.

Griffiths, C. E. M., \& Barker, J. N. W. N. (2010). Psoriasis. In D. Burns, B. SM \& C. N. e. al (Eds.), Rook's Textbook of Dermatology (8th ed.). Oxford: WileyBlackwell.

Griffiths, C. E. M., \& Richards, H. L. (2001). Psychological influences in psoriasis. Clinical and Experimental Dermatology, 26(4), 338-342.

Krueger, G., Koo, J., Lebwohl, M., Menter, A., Stern, R. S., \& Rolstad, T. (2001). The impact of psoriasis on quality of life: results of a 1998 National Psoriasis Foundation patient-membership survey. Arch Dermatol, 137(3), 280-284.

Langley, R. G., Krueger, G. G., \& Griffiths, C. E. (2005). Psoriasis: epidemiology, clinical features, and quality of life. Ann Rheum Dis, 64 Suppl 2, ii18-23; discussion ii24-15. doi: 64/suppl_2/ii18 [pii]

10.1136/ard.2004.033217 [doi]

Meyer, N., Paul, C., Feneron, D., Bardoulat, I., Thiriet, C., Camara, C., . . Ortonne, J. P. (2010). Psoriasis: an epidemiological evaluation of disease burden in 590 patients. Journal of the European Academy of Dermatology and Venereology, 24(9), 1075-1082. doi: 10.1111/j.1468-3083.2010.03600.x 
Poulin, Y., Papp, K. A., Wasel, N. R., Andrew, R., Fraquelli, E., Bernstein, G., \& Chan, D. (2010). A Canadian online survey to evaluate awareness and treatment satisfaction in individuals with moderate to severe plaque psoriasis. International Journal of Dermatology, 49(12), 1368-1375. doi: 10.1111/j.13654632.2010.04660.x

Schmitt-Egenolf, M. (2006). Psoriasis therapy in real life: The need for registries. Dermatology, 213(4), 327-330. doi: 10.1159/000096196

Seng, T. K., \& Nee, T. S. (1997). Group therapy: A useful and supportive treatment for psoriasis patients. International Journal of Dermatology, 36(2), 110-112.

Unaeze, J., Nijsten, T., Murphy, A., Ravichandran, C., \& Stern, R. S. (2006). Impact of psoriasis on health-related quality of life decreases over time: an 11-year prospective study. J Invest Dermatol, 126(7), 1480-1489. doi: 5700229 [pii]

10.1038/sj.jid.5700229 [doi]

Wahl, A. K., Mork, C., Lillehol, B. M., Myrdal, A. M., Helland, S., Hanestad, B. R., \& Moum, T. (2006). Changes in quality of life in persons with eczema and psoriasis after treatment in departments of dermatology. Acta Derm Venereol, 86(3), 198-201. doi: 10.2340/00015555-0062 [doi] 